\title{
On Quantum Mechanics on Noncommutative Quantum Phase Space
}

\author{
A.E.F. DJEMAÏ* \\ Abdus Salam International Centre for Theoretical Physics, 34100, Trieste, Italy \\ and \\ H. SMAIL \\ Département de Physique, Institut d'Hydraulique, \\ Centre Universitaire Mustapha Stambouli, Mascara, 29000, Algeria
}

August, 2003

\begin{abstract}
In this work, we develop a general framework in which Noncommutative Quantum Mechanics (NCQM), characterized by a space noncommutativity matrix parameter $\theta=\epsilon_{i j}{ }^{k} \theta_{k}$ and a momentum noncommutativity matrix parameter $\beta_{i j}=\epsilon_{i j}{ }^{k} \beta_{k}$, is showed to be equivalent to Quantum Mechanics (QM) on a suitable transformed Quantum Phase Space (QPS). Imposing some constraints on this particular transformation, we firstly find that the product of the two parameters $\theta$ and $\beta$ possesses a lower bound in direct relation with Heisenberg incertitude relations, and secondly that the two parameters are equivalent but with opposite sign, up to a dimension factor depending on the physical system under study. This means that noncommutativity is represented by a unique parameter which may play the role of a fundamental constant characterizing the whole NCQPS. Within our framework, we treat some physical systems on NCQPS : free particle, harmonic oscillator, system of two-charged particles, Hydrogen atom. Among the obtained results, we discover a new phenomenon which consists to see a free particle on NCQPS as equivalent to a harmonic oscillator with Larmor frequency depending on $\beta$, representing the same particle in presence of a magnetic field $\vec{B}=q^{-1} \vec{\beta}$. For the other examples, additional correction terms depending on $\beta$ appear in the expression of the energy spectrum. Finally, in the two-particle system case, we emphasize the fact that for two opposite charges noncommutativity is effectively perceived with opposite sign.
\end{abstract}

PACS : 03.65.ca and 11.10.Nx

Key words : Noncommutative space, Quantum Mechanics, Moyal product.

${ }^{*}$ Permanent address : Département de Physique, Faculté des Sciences, Université d'Oran Es-sénia, 31100, Oran, Algeria. Fax : (00).(213).(41).41.91.84, e-mail : abedelfarid@yahoo.com 


\section{Introduction}

Let $\mathbf{x}_{\mathbf{i}}, \mathbf{p}_{\mathbf{i}}$ be the position and momentum operators which generate the Heisenberg algebra of QM:

$$
\left[\mathbf{x}_{\mathbf{i}}, \mathbf{x}_{\mathbf{j}}\right]=0, \quad\left[\mathbf{x}_{\mathbf{i}}, \mathbf{p}_{\mathbf{j}}\right]=i \hbar \delta_{i j} \mathbf{1}, \quad\left[\mathbf{p}_{\mathbf{i}}, \mathbf{p}_{\mathbf{j}}\right]=0
$$

where the dynamics is described by the canonical equations :

$$
\dot{\mathbf{x}}_{\mathbf{i}}=\left[\mathbf{x}_{\mathbf{i}}, \mathbf{H}\right] \quad, \quad \dot{\mathbf{p}}_{\mathbf{i}}=\left[\mathbf{p}_{\mathbf{i}}, \mathbf{H}\right]
$$

with $\mathbf{H}$ being the Hamiltonian operator of the quantum system described on QPS.

In the context of "Quantization by deformation", [1], it has been shown that this operator algebra is equivalent to a $\hbar$-star deformation of the Poisson algebra of classical observables equipped with a Weyl-Wigner-Moyal product defined as follows:

$$
(f * \hbar g)(u)=\left.\exp \left[\frac{i \hbar}{2} \omega^{a b} \partial_{a}^{(1)} \partial_{b}^{(2)}\right] f\left(u_{1}\right) g\left(u_{2}\right)\right|_{u_{1}=u_{2}=u}
$$

where $u_{a}$ are phase space variables, $a=1, \ldots ., 2 N$, and $\omega$ is the usual symplectic structure .

Moreover, it has been shown in 2] that QM can be formulated as a noncommutative symplectic geometry by means of a discrete Weyl-Schwinger realization of the Heisenberg group and by developing a discrete version of the Weyl-Wigner-Moyal formalism. In analogy with the classical case, the noncommutative (quantum) symplectic geometry associated with the matrix algebra $M_{N}(\mathbf{C})$ generated by Schwinger basis was described.

Recently, there has been a growing interest on the description of QM on non-commutative spaces (NCQM), 3] - 6]. This was motivated by string theory arguments, which tell us that the low energy effective theory of D-brane in the background of NS-NS $B$ field lives on noncommutative spaces, [7. In fact, in this context, our space-time may be the worldvolume of a D-brane, and thus may be noncommutative. Moreover, it has been shown that the study of the dynamics of a quantum system on a noncommutative space is equivalent to the study of the dynamics of this system on a commutative space in presence of a magnetic field $\vec{B},[5]$.

A noncommutative space can be realized by coordinate operators satisfying :

$$
\left[\mathbf{x}_{\mu}, \mathbf{x}_{\nu}\right]_{\star}=i \hbar \theta_{\mu \nu}
$$

where $\theta_{\mu \nu}$ is the noncommutativity parameter of dimension $\frac{(\text { lenght })^{2}}{\hbar}$ represented by an antisymmetric matrix whose entries $\theta_{0 i}$ are considered to be vanishing, otherwise the theory is not unitary. Performing explicit loop calculations, it has been proved that, for instance, the NonCommutative QED (NCQED) up to one loop is renormalizable. Then, in order to study phenomenological consequences of the noncommutativity of the space, it is more indicated to try to learn more about the QM on NC spaces.

The aim of this work is to study QM on NCQPS. We find that, in addition to the results obtained in various papers where the noncommutativity is only present in the space sector, there are additional terms due to the noncommutativity $\beta$ present in the momentum sector of QPS since its existence is, in fact, due essentially to the existence of the noncommutativity $\theta$ on the space.

In this work, we construct a general $\alpha$-star deformation of the algebra of classical observables that gives rise to a general NCQM. It appears that this $\alpha$-star deformation is equivalent to some general transformation on the usual quantum phase space variables $\left(\mathbf{x}_{i}, \mathbf{p}_{i}\right)$. Indeed, we show 
that NCQM is equivalent to QM on a transformed QPS.

This paper is organized as follows. In section 2, we describe a general NCQM. The section 3 is devoted to study the noncommutative Hamiltonian dynamics of a quantum system. Some simple examples of quantum systems are considered like : free particle, harmonic oscillator, two-particle system and as a particular case the Hydrogen atom. Finally, section 4 is devoted to some conclusions and perspectives.

\section{Description of a general Noncommutative quantum Mechanics}

Let us consider a general $\alpha$-star deformation on the Poisson algebra of the classical observables as:

$$
\left(f *_{\alpha} g\right)(u)=\left.\exp \left[\frac{1}{2} \alpha_{a b} \partial_{a}^{(1)} \partial_{b}^{(2)}\right] f\left(u_{1}\right) g\left(u_{2}\right)\right|_{u_{1}=u_{2}=u}
$$

where $\alpha$ generalizes the usual classical symplectic structure $\omega$ and its general form may be given by:

$$
\alpha=\left(\begin{array}{cc}
\theta & \mathbf{1}+\sigma \\
-\mathbf{1}-\sigma & \beta
\end{array}\right)
$$

where $\theta$ and $\beta$ are antisymmetric $3 \times 3$ matrices and $\sigma$ a symmetric $3 \times 3$ matrix, such that:

$$
\theta_{i j}=\epsilon_{i j}^{k} \theta_{k} \quad, \quad \beta_{i j}=\epsilon_{i j}^{k} \beta_{k}
$$

The $\alpha$-star deformed Poisson algebra is given by:

$$
\left\{x_{i}, x_{j}\right\}_{\alpha}=\theta_{i j} \quad, \quad\left\{x_{i}, p_{j}\right\}_{\alpha}=\delta_{i j}+\sigma_{i j} \quad, \quad\left\{p_{i}, p_{j}\right\}_{\alpha}=\beta_{i j} .
$$

It is easy to see that the classical limit is guaranteed by the condition $\theta=\beta=\sigma=\mathbf{0}$.

Considering the noncommutativity as a small perturbation on the structure of the phase space, the real parameters $\theta_{i}$ and $\beta_{i}$ are taken very small and our calculations are taken up to first order in $\theta$ and $\beta$. We will see that $\sigma$ can be ignored since it is of second order.

The above star-product permits us to deduce the star deformed Heisenberg algebra which defines a general NCQM:

$$
\left[\mathbf{x}_{\mathbf{i}}, \mathbf{x}_{\mathbf{j}}\right]_{\alpha}=i \hbar \theta_{i j} \mathbf{1}, \quad\left[\mathbf{x}_{\mathbf{i}}, \mathbf{p}_{\mathbf{j}}\right]_{\alpha}=i \hbar\left(\delta_{i j}+\sigma_{i j}\right) \mathbf{1}, \quad\left[\mathbf{p}_{\mathbf{i}}, \mathbf{p}_{\mathbf{j}}\right]_{\alpha}=i \hbar \beta_{i j} \mathbf{1}
$$

via a generalized Dirac quantization :

$$
\{,\}_{\alpha} \longrightarrow \frac{1}{i \hbar}[,]_{\alpha}
$$

and with a noncommutative quantum dynamics governed by the motion equations:

$$
\dot{\mathbf{x}}_{i}=\left[\mathbf{x}_{i}, \mathbf{H}\right]_{\alpha} \quad, \quad \dot{\mathbf{p}}_{i}=\left[\mathbf{p}_{i}, \mathbf{H}\right]_{\alpha}
$$

Here, we argue that introducing a noncommutativity parametrized by $\theta$ on the space sector of the QPS, we automatically introduce a noncommutativity parametrized by another parameter, say $\beta$, on the momentum sector of QPS, since they are linked by the famous Heisenberg incertitude relations, and then, the parameter $\sigma$ which appears in the star-commutator of a space operator 
with a momentum operator must be tied to the two parameters $\theta$ and $\beta$. Hereunder, we will give a more precise explanation to this point.

Let us consider a general linear transformation on the usual quantum phase space variables:

$$
\left\{\begin{array}{l}
\mathbf{x}_{i}^{\prime}=a_{i k} \mathbf{x}_{k}+b_{i k} \mathbf{p}_{k} \\
\mathbf{p}_{i}^{\prime}=c_{i k} \mathbf{x}_{k}+d_{i k} \mathbf{p}_{k}
\end{array}\right.
$$

At this stage, we remark that for the following particular choice of the matrix parameters $a, b, c$, and $d$ :

$$
a=d=1 \quad, \quad c=\frac{1}{2} \beta \quad, \quad b=-\frac{1}{2} \theta
$$

the new quantum variables $\mathbf{x}_{i}^{\prime}$ and $\mathbf{p}_{i}^{\prime}$ :

$$
\left(\begin{array}{c}
\mathbf{x}_{i}^{\prime} \\
\mathbf{p}_{i}^{\prime}
\end{array}\right)=\left(\begin{array}{cc}
\delta_{i k} & -\frac{1}{2} \theta_{i k} \\
\frac{1}{2} \beta_{i k} & \delta_{i k}
\end{array}\right)\left(\begin{array}{c}
\mathbf{x}_{k} \\
\mathbf{p}_{k}
\end{array}\right)=T_{i k}\left(\begin{array}{c}
\mathbf{x}_{k} \\
\mathbf{p}_{k}
\end{array}\right)
$$

or, in another manner:

$$
\left\{\begin{array}{l}
\overrightarrow{\mathbf{x}}^{\prime}=\overrightarrow{\mathbf{x}}-\frac{1}{2} \overrightarrow{\mathbf{p}} \wedge \vec{\theta}=\overrightarrow{\mathbf{x}}-\frac{1}{2} \theta \overrightarrow{\mathbf{p}} \\
\overrightarrow{\mathbf{p}}^{\prime}=\overrightarrow{\mathbf{p}}+\frac{1}{2} \overrightarrow{\mathbf{x}} \wedge \vec{\beta}=\overrightarrow{\mathbf{p}}+\frac{1}{2} \beta \overrightarrow{\mathbf{x}}
\end{array}\right.
$$

satisfy commutation relations that look like (4) :

$$
\left[\mathbf{x}_{i}^{\prime}, \mathbf{x}_{j}^{\prime}\right]=i \hbar \theta_{i j} \mathbf{1},\left[\mathbf{x}_{i}^{\prime}, \mathbf{p}_{j}^{\prime}\right]=i \hbar\left(\delta_{i j}+\sigma_{i j}\right) \mathbf{1},\left[\mathbf{p}_{i}^{\prime}, \mathbf{p}_{j}^{\prime}\right]=i \hbar \beta_{i j} \mathbf{1}
$$

where $\sigma$ must satisfy the following condition :

$$
\sigma=-\frac{1}{8}(\theta \beta+\beta \theta)
$$

and so, it can be ignored since we are only interested by first order terms in $\theta$ and $\beta$.

Then, instead of using quantum variables with $*_{\alpha}$-product (see (44)), it is more suitable to use the transformed quantum variables $\left(\mathbf{x}_{i}^{\prime}, \mathbf{p}_{i}^{\prime}\right)$ with the usual operator product.

Investigating the transformation (6] ), we see that its Jacobian is given by:

$$
J=\operatorname{det}(T)=\left(1+\frac{1}{8} \rho\right)^{2} \neq 0
$$

where:

$$
\rho=\operatorname{tr}(\theta \cdot \beta)=\operatorname{tr}(\beta \cdot \theta)=-2 \vec{\theta} \cdot \vec{\beta}
$$

Imposing to $J$ to be equal to 1 , the parameter $\rho$ is constrained to fulfill the following condition:

$$
\rho=-16 \Longrightarrow \vec{\theta} \cdot \vec{\beta}=8
$$

From the relations (77), we see that the dimension of $\theta_{i}$ is the same as $\frac{\left(\Delta x_{i}\right)^{2}}{\hbar}$ and the dimension of $\beta_{i}$ as $\frac{\left(\triangle p_{i}\right)^{2}}{\hbar}$ and then the above condition looks like the well known Heisenberg incertitude relations :

$$
\Delta x_{i} . \Delta p_{i} \geq \hbar
$$


It follows that the noncommutative perturbation along the space-like and momentum-like sectors must verify the same kind of incertitude relations as do the position and the momentum operators. The right side of the equation (8) gives in fact the lower bound of the product of the two parameters $\theta$ and $\beta$.

Furthermore, imposing to the matrix transformation $T$ to be orthogonal :

$$
T T^{t}=T^{t} T=\mathbf{1}
$$

we deduce that the deformation parameters $\theta_{i}$ in the space-like sector of the quantum phase space are of the same "magnitude", up to a dimension factor depending on the physical system under study, as the deformation parameters $\beta_{i}$ in the momentum-like sector:

$$
\theta_{i} \sim-\beta_{i}
$$

Effectively, this special orthogonal transformation on the quantum phase space expresses that, if one introduces a noncommutativity as a perturbation on the space like sector of QPS, this leads automatically to introduce a noncommutativity as an opposite equivalent perturbation on the momentum-like sector. In fact, $T$ looks like an infinitesimal transformation on QPS :

$$
T=\left(\begin{array}{ll}
\mathbf{1} & \mathbf{0} \\
\mathbf{0} & \mathbf{1}
\end{array}\right)-\frac{1}{2}\left(\begin{array}{cc}
\mathbf{0} & \theta \\
-\beta & \mathbf{0}
\end{array}\right)
$$

Hereunder, we will show that noncommutative effects are always tied to the angular momentum of the system under study and so, they emphasize the presence of some kind of rotations relatively to the axis defined by $\vec{\theta}$.

\section{Noncommutative Quantum Dynamics}

In this section we investigate some examples of dynamical systems on NCQPS within the framework presented in the previous section. In particular, we propose to treat the cases of a free particle, a harmonic oscillator, two-particle system and in particular Hydrogen atom.

Let us begin to investigate the general case of a quantum system with a Hamiltonian operator :

$$
H(\overrightarrow{\mathbf{x}}, \overrightarrow{\mathbf{p}})=\frac{\overrightarrow{\mathbf{p}}^{2}}{2 m}+V(\overrightarrow{\mathbf{x}})
$$

where $\overrightarrow{\mathbf{x}}$ and $\overrightarrow{\mathbf{p}}$ satisfy (11). Its quantum dynamics is governed by the motion equation (2).

We have shown that the study of this quantum system on a NCQPS where the position and momentum operators obey the nontrivial commutation relations (4) is equivalent to study it on the usual QPS subject to the transformation (6), where the new position and momentum operators $\mathbf{x}^{\prime}$ and $\mathbf{p}^{\prime}$ obey the commutation relations (17).

In this context, the Hamiltonian operator varies as :

$$
\Delta H=H\left(\overrightarrow{\mathbf{x}}^{\prime}, \overrightarrow{\mathbf{p}}^{\prime}\right)-H(\overrightarrow{\mathbf{x}}, \overrightarrow{\mathbf{p}})=-\frac{1}{2 m} \beta_{i k} \mathbf{x}_{i} \mathbf{p}_{k}+\left[V\left(\overrightarrow{\mathbf{x}}^{\prime}\right)-V(\overrightarrow{\mathbf{x}})\right]=-\frac{1}{2 m} \overrightarrow{\mathbf{L}} \cdot \vec{\beta}+\Delta V
$$

where $\overrightarrow{\mathbf{L}}=\overrightarrow{\mathbf{x}} \wedge \overrightarrow{\mathbf{p}}$ is the angular momentum of the quantum system. It is clear that there exists a correction term $\Delta E_{N C}$ to the energy spectrum of our quantum dynamical system due to the presence of noncommutativity in the QPS reflected by the two additional terms in the right side of the equation (9). 


\subsection{Free Particle}

In the case of a quantum free particle $(V(\overrightarrow{\mathbf{x}})=\mathbf{0})$, the Hamiltonian transforms as (see (9) ) :

$$
H^{\prime}(\overrightarrow{\mathbf{x}}, \overrightarrow{\mathbf{p}})=\frac{\overrightarrow{\mathbf{p}}^{\prime 2}}{2 m}=\frac{\overrightarrow{\mathbf{p}}^{2}}{2 m}-\frac{1}{2 m} \beta_{i k} \mathbf{x}_{i} \mathbf{p}_{k}=\frac{\overrightarrow{\mathbf{p}}^{2}}{2 m}-\frac{1}{2 m} \overrightarrow{\mathbf{L}} \cdot \vec{\beta}
$$

where:

$$
\overrightarrow{\mathbf{p}}^{\prime}=\overrightarrow{\mathbf{p}}+\frac{1}{2}(\overrightarrow{\mathbf{x}} \wedge \vec{\beta})
$$

This shows that the study of the dynamics of a particle on a NCQPS is equivalent to the study of the dynamics of this particle of charge $q$ on the usual QPS in presence of a magnetic field. This interpretation is justified by the identification of the additional term in (10) as a vector potential $\overrightarrow{\mathbf{A}}$ associated with a magnetic field $\vec{B}$ :

$$
q \overrightarrow{\mathbf{A}}=-\frac{1}{2} \overrightarrow{\mathbf{x}} \wedge \vec{\beta} \quad / \quad \vec{\beta}=q \vec{B}
$$

Considering the simple case $\beta_{1}=\beta_{2}=0$ and $\beta_{3}=\beta=q B$, and defining :

$$
\mathbf{z}=\sqrt{\frac{|\beta|}{2 \hbar}}(\mathbf{x}+i \mathbf{y})
$$

and the annihilation and creation operators :

$$
\mathbf{a}=\partial_{\bar{z}}+\frac{1}{2} \mathbf{z} \quad, \quad \mathbf{a}^{+}=-\partial_{z}+\frac{1}{2} \overline{\mathbf{z}}
$$

one finds that our quantum particle looks like a harmonic oscillator system :

$$
[\mathbf{a}, \mathbf{a}]=\left[\mathbf{a}^{+}, \mathbf{a}^{+}\right]=\mathbf{0} \quad, \quad\left[\mathbf{a}, \mathbf{a}^{+}\right]=\mathbf{1}
$$

with Hamiltonian operator :

$$
H\left(\mathbf{a}, \mathbf{a}^{+}\right)=\hbar \omega_{\beta}\left(\mathbf{a}^{+} \mathbf{a}+\frac{1}{2} \mathbf{1}\right)
$$

giving us a spectrum formed by infinite degenerate Landau levels :

$$
E_{l}=\hbar \omega_{\beta}\left(l+\frac{1}{2}\right) \quad, \quad l \in \mathbf{Z}
$$

where

$$
\omega_{\beta}=\frac{|\beta|}{m}=\frac{|q B|}{m}
$$

denotes the Larmor frequency.

Finally, we conclude that introducing a noncommutativity on the QPS, the free quantum particle behaves as a harmonic oscillator with a (very small) frequency depending on the noncommutative perturbation $\beta$ on the momentum sector. 


\subsection{Harmonic oscillator}

Let us consider now the example of a quantum harmonic oscillator of charge $q$ and with a potential :

$$
V(\overrightarrow{\mathbf{x}})=\frac{1}{2} \kappa \overrightarrow{\mathbf{x}}^{2}
$$

and let set :

$$
\vec{\mu}=[\vec{\beta}+m \kappa \vec{\theta}] .
$$

Then, the modified Hamiltonian becomes (see (9)) :

$$
H^{\prime}(\overrightarrow{\mathbf{x}}, \overrightarrow{\mathbf{p}})=H(\overrightarrow{\mathbf{x}}, \overrightarrow{\mathbf{p}})-\frac{1}{2 m} \overrightarrow{\mathbf{L}} \cdot \vec{\mu}=\frac{\overrightarrow{\mathbf{P}}^{2}}{2 m}+V(\overrightarrow{\mathbf{x}})
$$

where:

$$
\overrightarrow{\mathbf{P}}=\overrightarrow{\mathbf{p}}+\frac{1}{2} \overrightarrow{\mathbf{x}} \wedge \vec{\mu}
$$

The resulting system is then always a quantum harmonic oscillator but with a shifted energy spectrum. In fact, it looks also as the ordinary harmonic oscillator in presence of a magnetic field :

$$
\vec{B}=q^{-1} \vec{\mu}=q^{-1}[\vec{\beta}+m \kappa \vec{\theta}]
$$

which is the sum of two magnetic fields $\vec{B}_{1}=q^{-1} \vec{\beta}$ and $\vec{B}_{2}=q^{-1} m \kappa \vec{\theta}$ due to the presence of noncommutativity in the momentum and space sectors of QPS respectively.

The new Larmor frequency characterizing the correction term in the system spectrum is :

$$
\omega_{\mu}=\frac{|\mu|}{m}=\frac{|q B|}{m} .
$$

\subsection{Two-particle system}

Let us treat now a system of two distinct quantum particles with respective masses and charges $\left(m_{a}, q_{a}\right)$ and $\left(m_{b}, q_{b}\right)$, defined on NCQPS. Moreover, since the dynamical states of two different particles belong to two different Hilbert spaces, one considers that their position and momentum operators commute under any product, stared or not. This leads to the following set of nontrivial commutation relations :

$$
\begin{gathered}
{\left[\hat{\mathbf{x}}_{i}^{(a)}, \hat{\mathbf{x}}_{j}^{(a)}\right]_{\star}=i \hbar \theta_{i j}^{(a)}, \quad\left[\hat{\mathbf{x}}_{i}^{(a)}, \hat{\mathbf{p}}_{j}^{(a)}\right]_{\star}=i \hbar\left(\delta_{i j}+\sigma_{i j}\right),\left[\hat{\mathbf{p}}_{i}^{(a)}, \hat{\mathbf{p}}_{j}^{(a)}\right]_{\star}=i \hbar \beta_{i j}^{(a)}} \\
{\left[\hat{\mathbf{x}}_{i}^{(b)}, \hat{\mathbf{x}}_{j}^{(b)}\right]_{\star}=i \hbar \theta_{i j}^{(b)}, \quad\left[\hat{\mathbf{x}}_{i}^{(b)}, \hat{\mathbf{p}}_{j}^{(b)}\right]_{\star}=i \hbar\left(\delta_{i j}+\sigma_{i j}\right),\left[\hat{\mathbf{p}}_{i}^{(b)}, \hat{\mathbf{p}}_{j}^{(b)}\right]_{\star}=i \hbar \beta_{i j}^{(b)}} \\
{\left[\hat{\mathbf{x}}_{i}^{(a)}, \hat{\mathbf{x}}_{j}^{(b)}\right]_{\star}=\left[\hat{\mathbf{x}}_{i}^{(a)}, \hat{\mathbf{p}}_{j}^{(b)}\right]_{\star}=\left[\hat{\mathbf{p}}_{i}^{(a)}, \hat{\mathbf{x}}_{j}^{(b)}\right]_{\star}=\left[\hat{\mathbf{p}}_{i}^{(a)}, \hat{\mathbf{p}}_{j}^{(b)}\right]_{\star}=\mathbf{0}}
\end{gathered}
$$

Within our framework, the study of this two-particle system on NCQPS is equivalent to study it on the usual QPS on which we perform the transformation (6), where $\sigma$ can be ignored since it is of second order. This means that we have the new variables :

$$
\mathbf{x}_{i}^{(a)}=\mathbf{x}_{i}^{(a)}-\frac{1}{2} \theta_{i j}^{(a)} \mathbf{p}_{j}^{(a)} \quad, \quad \mathbf{p}_{i}^{,(a)}=\mathbf{p}_{i}^{(a)}+\frac{1}{2} \beta_{i j}^{(a)} \mathbf{x}_{j}^{(a)}
$$




$$
\mathbf{x}_{i}^{(b)}=\mathbf{x}_{i}^{(b)}-\frac{1}{2} \theta_{i j}^{(b)} \mathbf{p}_{j}^{(b)} \quad, \quad \mathbf{p}_{i}^{(b)}=\mathbf{p}_{i}^{(b)}+\frac{1}{2} \beta_{i j}^{(b)} \mathbf{x}_{j}^{(b)}
$$

where the "primed" variables obey the same commutation relations (12), (13) and (14) as do the noncommutative variables, but without $\star$-product. The non-primed variables $\mathbf{x}_{i}^{(a, b)}$ and $\mathbf{p}_{i}^{(a, b)}$ generate usual Heisenberg algebras for each particle.

To deal with the two-particle system, let us consider the following more convenient set of operators :

$$
\begin{array}{rlr}
\mathbf{X}_{i} & =\mathbf{x}_{i}^{(a)}-\mathbf{x}_{i}^{(b)} & \text { "relative coordinate operators" } \\
\mathbf{Y}_{i} & =\frac{m_{a} \mathbf{x}_{i}^{(a)}+m_{b} \mathbf{x}_{i}^{(b)}}{m_{a}+m_{b}} \quad \text { "center of mass coordinate operator s" } \\
\mathbf{P}_{i} & =\mathbf{p}_{i}^{(a)}+\mathbf{p}^{(b)} & \text { "total momentum operators" } \\
\mathbf{Q}_{i} & =\frac{m_{b} \mathbf{p}_{i}^{(a)}-m_{a} \mathbf{p}_{i}^{(b)}}{m_{a}+m_{b}} \quad \text { "relative momentum operators" }
\end{array}
$$

and let us introduce :

$$
\begin{array}{rlr}
M & =m_{a}+m_{b} & \text { "total mass" } \\
\mu & =\frac{m_{a} m_{b}}{m_{a}+m_{b}} & \text { "reduced mass" }
\end{array}
$$

These new two-particle system operators verify the following set of commutation relations:

$$
\left[\mathbf{X}_{i}, \mathbf{Q}_{j}\right]=\left[\mathbf{Y}_{i}, \mathbf{P}_{j}\right]=i \hbar \delta_{i j}
$$

and all the others are vanishing.

The primed corresponding operators satisfy :

$$
\begin{aligned}
& {\left[\mathbf{X}_{i}^{\prime}, \mathbf{X}_{j}^{\prime}\right]=i \hbar\left(\theta_{i j}^{(a)}+\theta_{i j}^{(b)}\right) \mathbf{1}, \quad\left[\mathbf{X}_{i}^{\prime}, \mathbf{Y}^{\prime}{ }_{j}\right]=i \hbar\left(\frac{m_{a}}{M} \theta_{i j}^{(a)}-\frac{m_{b}}{M} \theta_{i j}^{(b)}\right) \mathbf{1}} \\
& {\left[\mathbf{X}_{i}^{\prime}, \mathbf{P}^{\prime}{ }_{j}\right]=\mathbf{0},\left[\mathbf{X}_{i}^{\prime}, \mathbf{Q}_{j}^{\prime}\right]=i \hbar \delta_{i j} \mathbf{1}} \\
& {\left[\mathbf{Y}_{i}^{\prime}, \mathbf{Y}^{\prime}{ }_{j}\right]=i \hbar\left(\frac{m_{a}^{2}}{M^{2}} \theta_{i j}^{(a)}+\frac{m_{b}^{2}}{M^{2}} \theta_{i j}^{(b)}\right) \mathbf{1}, \quad\left[\mathbf{Y}_{i}^{\prime}, \mathbf{P}^{\prime}{ }_{j}\right]=i \hbar \delta_{i j} \mathbf{1}} \\
& {\left[\mathbf{Y}_{i}^{\prime}, \mathbf{Q}_{j}^{\prime}\right]=\mathbf{0}, \quad\left[\mathbf{P}_{i}^{\prime}, \mathbf{P}^{\prime}{ }_{j}\right]=i \hbar\left(\beta_{i j}^{(a)}+\beta_{i j}^{(b)}\right) \mathbf{1}} \\
& {\left[\mathbf{P}_{i}^{\prime}, \mathbf{Q}_{j}^{\prime}\right]=i \hbar\left(\frac{m_{b}}{M} \beta_{i j}^{(a)}-\frac{m_{a}}{M} \beta_{i j}^{(b)}\right) \mathbf{1}, \quad\left[\mathbf{Q}_{i}^{\prime}, \mathbf{Q}_{j}^{\prime}\right]=i \hbar\left(\frac{m_{b}^{2}}{M^{2}} \beta_{i j}^{(a)}+\frac{m_{a}^{2}}{M^{2}} \beta_{i j}^{(b)}\right) \mathbf{1}}
\end{aligned}
$$

Under our transformation, the Hamiltonian of this two-particle system :

$$
H\left(\overrightarrow{\mathbf{x}}^{(a)}, \overrightarrow{\mathbf{x}}^{(b)}, \overrightarrow{\mathbf{p}}^{(a)}, \overrightarrow{\mathbf{p}}^{(b)}\right)=\frac{\mathbf{p}_{i}^{(a)} \mathbf{p}^{(a) i}}{2 m_{a}}+\frac{\mathbf{p}_{i}^{(b)} \mathbf{p}^{(b) i}}{2 m_{b}}+V\left(\overrightarrow{\mathbf{x}}^{(a)}, \overrightarrow{\mathbf{x}}^{(b)}\right)
$$

or, in terms of the new (non-primed) variables :

$$
H(\overrightarrow{\mathbf{X}}, \overrightarrow{\mathbf{Y}}, \overrightarrow{\mathbf{P}}, \overrightarrow{\mathbf{Q}})=\frac{\mathbf{P}_{i} \mathbf{P}^{i}}{2 M}+\frac{\mathbf{Q}_{i} \mathbf{Q}^{i}}{2 \mu}+V(\overrightarrow{\mathbf{X}}, \overrightarrow{\mathbf{Y}})
$$

will change like :

$$
H\left({\overrightarrow{\mathbf{x}^{\prime}}}^{(a)},{\overrightarrow{\mathbf{x}^{\prime}}}^{(b)},{\overrightarrow{\mathbf{p}^{\prime}}}^{(a)},{\overrightarrow{\mathbf{p}^{\prime}}}^{(b)}\right)=\frac{{\overrightarrow{\mathbf{p}^{\prime}}}_{i}^{(a)} \mathbf{p}^{\prime(a) i}}{2 m_{a}}+\frac{\mathbf{p}_{i}^{\prime(b)} \mathbf{p}^{\prime(b) i}}{2 m_{b}}+V\left({\overrightarrow{\mathbf{x}^{\prime}}}^{(a)},{\overrightarrow{\mathbf{x}^{\prime}}}^{(b)}\right)
$$


or equivalently :

$$
H\left(\overrightarrow{\mathbf{X}}^{\prime}, \overrightarrow{\mathbf{Y}}^{\prime}, \overrightarrow{\mathbf{P}}^{\prime}, \overrightarrow{\mathbf{Q}}^{\prime}\right)=\frac{\mathbf{P}_{i}^{\prime} \mathbf{P}^{\prime i}}{2 M}+\frac{\mathbf{Q}_{i}^{\prime} \mathbf{Q}^{\prime i}}{2 \mu}+V\left(\overrightarrow{\mathbf{X}}^{\prime}, \overrightarrow{\mathbf{Y}}^{\prime}\right)
$$

where :

$$
\begin{aligned}
\mathbf{X}_{i}{ }_{i} & =\mathbf{X}_{i}-\frac{1}{2} \theta_{i j}^{(a)} \mathbf{p}_{j}^{(a)}+\frac{1}{2} \theta_{i j}^{(b)} \mathbf{p}_{j}^{(b)} \\
\mathbf{Y}^{\prime}{ }_{i} & =\mathbf{Y}_{i}-\frac{m_{a}}{2 M} \theta_{i j}^{(a)} \mathbf{p}_{j}^{(a)}-\frac{m_{b}}{2 M} \theta_{i j}^{(b)} \mathbf{p}_{j}^{(b)} \\
\mathbf{P}^{\prime}{ }_{i} & =\mathbf{P}_{i}+\frac{1}{2} \beta_{i j}^{(a)} \mathbf{x}_{j}^{(a)}+\frac{1}{2} \beta_{i j}^{(b)} \mathbf{x}_{j}^{(b)} \\
\mathbf{Q}^{\prime}{ }_{i} & =\mathbf{Q}_{i}+\frac{m_{b}}{2 M} \beta_{i j}^{(a)} \mathbf{x}_{j}^{(a)}-\frac{m_{a}}{2 M} \beta_{i j}^{(b)} \mathbf{x}_{j}^{(b)}
\end{aligned}
$$

At this level, we recall our assumption presented in the precedent section which consists to consider that, up to a dimension factor depending on the physical system under study, we have for the same particle :

$$
\beta_{i j}^{(a)}=-\theta_{i j}^{(a)} \quad, \quad \beta_{i j}^{(b)}=-\theta_{i j}^{(b)}
$$

Furthermore, considering the particular and very interesting case of two-particle system whose charges $q_{a}$ and $q_{b}$ have opposite signs, and knowing that noncommutativity means the existence of a magnetic field on the QPS in presence of which charges of opposite signs have opposite motions, we can also argue that each of our two particles perceive the same noncommutativity but with opposite sign, [8] :

$$
\theta_{i j}^{(a)}=-\theta_{i j}^{(b)}=\theta_{i j} \quad, \quad \beta_{i j}^{(a)}=-\beta_{i j}^{(b)}=\beta_{i j}
$$

and so, finally, we are dealing with only one noncommutativity parameter which characterizes the whole NCQPS :

$$
\theta_{i j}=\theta_{i j}^{(a)}=-\theta_{i j}^{(b)}=\beta_{i j}^{(b)}=-\beta_{i j}^{(a)}
$$

In this case, the relations (17) reduce to :

$$
\begin{aligned}
\mathbf{X}_{i}^{\prime} & =\mathbf{X}_{i}-\frac{1}{2} \theta_{i j} \mathbf{P}_{j} \\
\mathbf{Y}^{\prime}{ }_{i} & =\mathbf{Y}_{i}-\frac{1}{2} \theta_{i j}\left[\mathbf{Q}_{j}+\left(\frac{m_{a}-m_{b}}{M}\right) \mathbf{P}_{j}\right] \\
\mathbf{P}^{\prime}{ }_{i} & =\mathbf{P}_{i}-\frac{1}{2} \theta_{i j} \mathbf{X}_{j} \\
\mathbf{Q}^{\prime} & =\mathbf{Q}_{i}-\frac{1}{2} \theta_{i j}\left[\mathbf{Y}_{j}-\left(\frac{m_{a}-m_{b}}{M}\right) \mathbf{X}_{j}\right]
\end{aligned}
$$

and the relations (15) become:

$$
\begin{aligned}
{\left[\mathbf{X}_{i}^{\prime}, \mathbf{Y}_{j}^{\prime}\right] } & =-\left[\mathbf{P}_{i}^{\prime}, \mathbf{Q}_{j}^{\prime}\right]=i \hbar \theta_{i j} \mathbf{1} \\
{\left[\mathbf{X}_{i}^{\prime}, \mathbf{Q}_{j}^{\prime}\right] } & =\left[\mathbf{Y}_{i}^{\prime}, \mathbf{P}_{j}^{\prime}\right]=i \hbar \delta_{i j} \mathbf{1} \\
{\left[\mathbf{Y}_{i}^{\prime}, \mathbf{Y}_{j}^{\prime}\right] } & =\left[\mathbf{Q}_{i}^{\prime}, \mathbf{Q}^{\prime}{ }_{j}\right]=i \hbar\left(\frac{m_{a}-m_{b}}{M}\right) \theta_{i j} \mathbf{1} \\
{\left[\mathbf{X}_{i}^{\prime}, \mathbf{X}_{j}^{\prime}\right] } & =\left[\mathbf{X}_{i}^{\prime}, \mathbf{P}_{j}^{\prime}\right]=\left[\mathbf{Y}_{i}^{\prime}, \mathbf{Q}_{j}^{\prime}\right]=\left[\mathbf{P}_{i}^{\prime}, \mathbf{P}^{\prime}{ }_{j}\right]=\mathbf{0}
\end{aligned}
$$


With these simplifications, the transformed Hamiltonian (16) will differ from the non-transformed one by :

$$
\begin{aligned}
\Delta H & =-\frac{1}{2 M} \theta_{i j} \mathbf{X}_{j} \mathbf{P}_{i}-\frac{1}{2 \mu} \theta_{i j} \mathbf{Q}_{i} \mathbf{Y}_{j}-\left(\frac{m_{b}-m_{a}}{2 m_{a} m_{b}}\right) \theta_{i j} \mathbf{Q}_{i} \mathbf{X}_{j}+V\left(\mathbf{X}^{\prime}, \mathbf{Y}^{\prime}\right)-V(\mathbf{X}, \mathbf{Y}) \\
& =\left[\frac{1}{2 M}(\overrightarrow{\mathbf{X}} \wedge \overrightarrow{\mathbf{P}})+\frac{1}{2 \mu}(\overrightarrow{\mathbf{Y}} \wedge \overrightarrow{\mathbf{Q}})+\left(\frac{m_{b}-m_{a}}{2 m_{a} m_{b}}\right)(\overrightarrow{\mathbf{X}} \wedge \overrightarrow{\mathbf{Q}})\right] \cdot \vec{\theta}+\Delta V
\end{aligned}
$$

which reduces to :

$$
\Delta H=\left[\frac{1}{2 m_{a}} \overrightarrow{\mathbf{L}}^{(a)}-\frac{1}{2 m_{b}} \overrightarrow{\mathbf{L}}^{(b)}\right] \cdot \vec{\theta}+\Delta V
$$

where :

$$
\overrightarrow{\mathbf{L}}^{(a)}=\overrightarrow{\mathbf{x}}^{(a)} \wedge \overrightarrow{\mathbf{p}}^{(a)} \quad, \quad \overrightarrow{\mathbf{L}}^{(b)}=\overrightarrow{\mathbf{x}}^{(b)} \wedge \overrightarrow{\mathbf{p}}^{(b)}
$$

are the usual momentum operators of the two particles "a" and "b".

Let us discuss now the dynamical states of the two-particle system represented by a collective wave function solution of the NC Schrödinger equation, [3], [5] :

$$
i \hbar \frac{\partial}{\partial t} \Psi\left(\vec{x}^{(a)}, \vec{x}^{(b)}, t\right)=\left[-\frac{\hbar^{2}}{2 m_{a}} \Delta^{(a)}-\frac{\hbar^{2}}{2 m_{b}} \Delta^{(b)}+V\left(\vec{x}^{(a)}, \vec{x}^{(b)}\right)\right] \star \Psi\left(\vec{x}^{(a)}, \vec{x}^{(b)}, t\right)
$$

where the generalized $\star$-product is defined by :

$$
\begin{aligned}
(f \star g)\left(\vec{x}^{(a)}, \vec{x}^{(b)}\right)=\exp \left\{i \frac{\hbar}{2}\left(\theta_{i j}^{(a)} \frac{\partial}{\partial y_{i}^{(a)}} \frac{\partial}{\partial z_{j}^{(a)}}+\theta_{i j}^{(b)} \frac{\partial}{\partial y_{i}^{(b)}} \frac{\partial}{\partial z_{j}^{(b)}}\right)\right\} \times \\
f\left(\vec{y}^{(a)}, \vec{y}^{(b)}\right) g\left(\vec{z}^{(a)}, \vec{z}^{(b)}\right) \mid\left\{\begin{array}{l}
\vec{y}^{(a)}=\vec{z}^{(a)}=\vec{x}^{(a)} \\
\vec{y}^{(b)}=\vec{z}^{(b)}=\vec{x}^{(b)}
\end{array}\right.
\end{aligned}
$$

Let us recall that the above relations concern "hated" variables. Instead to use these variables and following our approach, it is more suitable to use our "primed" variables which verify the same commutation relations as do the first ones but without $\star$-product. It follows that the NC Schrödinger equation reads now as :

$$
i \hbar \frac{\partial}{\partial t} \Psi\left({\overrightarrow{x^{\prime}}}^{(a)},{\overrightarrow{x^{\prime}}}^{(b)}, t\right)=\left[-\frac{\hbar^{2}}{2 m_{a}} \Delta^{\prime(a)}-\frac{\hbar^{2}}{2 m_{b}} \Delta^{\prime(b)}+V\left({\overrightarrow{x^{\prime}}}^{(a)},{\overrightarrow{x^{\prime}}}^{(b)}\right)\right] \Psi\left({\overrightarrow{x^{\prime}}}^{(a)},{\overrightarrow{x^{\prime}}}^{(b)}, t\right)
$$

or equivalently,

$$
i \hbar \frac{\partial}{\partial t} \Psi\left(\overrightarrow{X^{\prime}}, \overrightarrow{Y^{\prime}}, t\right)=\left[-\frac{\hbar^{2}}{2 \mu} \Delta_{X^{\prime}}-\frac{\hbar^{2}}{2 M} \Delta_{Y^{\prime}}+V\left(\overrightarrow{X^{\prime}}, \overrightarrow{Y^{\prime}}\right)\right] \Psi\left(\overrightarrow{X^{\prime}}, \overrightarrow{Y^{\prime}}, t\right)
$$

To solve this equation, we use the technique of separation of variables :

$$
\Psi\left(X^{\prime}, Y^{\prime}, t\right)=\varphi\left(X^{\prime}\right) \phi\left(Y^{\prime}\right) \exp \left[-i \frac{E^{\prime}}{\hbar} t\right]
$$

It results two separated differential equations concerning the functions $\varphi\left(X^{\prime}\right)$ and $\phi\left(Y^{\prime}\right)$. Solving the second one, one obtains :

$$
\phi\left(Y^{\prime}\right)=\exp \left[i \overrightarrow{K^{\prime}} \cdot \vec{Y}^{\prime}\right]
$$


where $\vec{K}$ play the role of a wave vector associated to the collective wave function relatively to the center of mass and $\vec{K}^{\prime}$ is its transformed. The second equation reads :

$$
\Delta_{X^{\prime}} \varphi\left(\vec{X}^{\prime}\right)+\frac{2 \mu}{\hbar^{2}}\left[E^{\prime}-V\left(\overrightarrow{X^{\prime}}, \overrightarrow{Y^{\prime}}\right)-\frac{\hbar^{2}}{2 M}\left|\overrightarrow{K^{\prime}}\right|^{2}\right] \varphi\left(\vec{X}^{\prime}\right)=0
$$

where :

$$
\begin{aligned}
\vec{X}^{\prime} & =\vec{X}+\frac{1}{2} \vec{\theta} \wedge \vec{P} \\
\vec{Y}^{\prime} & =\vec{Y}+\frac{1}{2}\left[\vec{\theta} \wedge \vec{Q}+\left(\frac{m_{a}-m_{b}}{M}\right) \vec{\theta} \wedge \vec{P}\right] \\
E^{\prime} & =E+\Delta E_{N C} \\
\Delta V & =V\left(\vec{X}^{\prime}, \vec{Y}^{\prime}\right)-V(\vec{X}, \vec{Y})
\end{aligned}
$$

Knowing that the usual two-particle Schrödinger equation reads :

$$
\Delta_{X} \varphi(\vec{X})+\frac{2 \mu}{\hbar^{2}}\left[E-V(\vec{X}, \vec{Y})-\frac{\hbar^{2}}{2 M}|\vec{K}|^{2}\right] \varphi(\vec{X})=0
$$

and using :

$$
\Delta_{X^{\prime}} \varphi\left(\vec{X}^{\prime}\right)=\left(\Delta_{X^{\prime}}+\Delta_{P^{\prime}}\right) \varphi\left(\vec{X}^{\prime}\right)=\left(\Delta_{X}+\Delta_{P}\right) \varphi\left(\vec{X}+\frac{1}{2} \vec{\theta} \wedge \vec{P}\right) \simeq \Delta_{X} \varphi\left(\vec{X}+\frac{1}{2} \vec{\theta} \wedge \vec{P}\right)
$$

and considering the following separation of variables :

$$
\varphi\left(\vec{X}^{\prime}\right)=\varphi(\vec{X}) F(\vec{\theta} \wedge \vec{P})
$$

we find that the noncommutative correction to the energy spectrum of this two-particle system is given by :

$$
\Delta E_{N C}=\Delta V-\frac{\hbar^{2}}{2 M}\left[\left|\overrightarrow{K^{\prime}}\right|^{2}-|\vec{K}|^{2}\right] .
$$

In fact, it is easy to see that this relation is another equivalent version of the relation (18).

\subsection{Hydrogen atom}

This example can be treated in two different manners. The first consists to consider this system as a one-particle system (Electron) in an external Coulomb potential (Nucleus). In this approach, we can directly use the relation (9) to deduce the $\mathrm{NC}$ correction of the Hamiltonian by injecting the potential :

$$
V(\overrightarrow{\mathbf{x}})=-\frac{Z e^{2}}{\sqrt{\mathbf{x}_{i} \mathbf{x}^{i}}}
$$

The second method consists to consider it as a two-particle system whose treatment is developed in the previous subsection.

Using the first method, we find that the variation of the potential reads :

$$
\Delta V=-\frac{Z e^{2}}{2 \mathbf{x}^{3}} \overrightarrow{\mathbf{L}} \cdot \vec{\theta}=-\frac{e}{2}(\vec{\theta} \wedge \overrightarrow{\mathbf{p}}) \cdot\left(\frac{-Z e \overrightarrow{\mathbf{x}}}{\mathbf{x}^{3}}\right)
$$


where $\mathbf{x}=\sqrt{\mathbf{x}_{i} \mathbf{x}^{i}}$ and then, the NC correction of the Hamiltonian is given by :

$$
\Delta H=\frac{1}{2 m}(\vec{\beta} \wedge \overrightarrow{\mathbf{p}}) \cdot \overrightarrow{\mathbf{x}}-\frac{e}{2}(\vec{\theta} \wedge \overrightarrow{\mathbf{p}}) \cdot\left(\frac{-Z e \overrightarrow{\mathbf{x}}}{\mathbf{x}^{3}}\right)=-\frac{1}{2 m} \overrightarrow{\mathbf{L}} \cdot \vec{\eta}
$$

where

$$
\vec{\eta}=\vec{\beta}+\frac{m Z e^{2}}{\mathbf{x}^{3}} \vec{\theta}
$$

The first term in $\Delta H$ corresponds to the existence of a magnetic field as already obtained in the precedent cases. It comes as a kinetic correction term. The second one that comes as a potential correction term looks like a spin-orbit coupling term where the noncommutativity induced spin momentum $\vec{S}$ is given by :

$$
\vec{S}=\frac{\hbar}{\lambda_{e}^{2}} \vec{\theta}
$$

where $\lambda_{e}$ is the usual Compton wave length of the electron, [3].

Following the second approach, we have first to calculate $\Delta V$ (see (18)) :

$$
\Delta V=-\frac{e}{2}(\vec{\theta} \wedge \overrightarrow{\mathbf{P}}) \cdot\left(\frac{-Z e^{2} \overrightarrow{\mathbf{X}}}{\overrightarrow{\mathbf{X}}}\right)=-\frac{Z e^{2}}{2 \mathbf{X}^{3}} \overrightarrow{\mathbf{E}} \cdot \vec{\theta}
$$

where $\mathbf{X}=\sqrt{\mathbf{X}_{i} \mathbf{X}^{i}}$ and $\overrightarrow{\mathbf{E}}=\overrightarrow{\mathbf{X}} \wedge \overrightarrow{\mathbf{P}}$.

Then, the NC correction to the Hamiltonian is now given by :

$$
\Delta H=\left[\frac{1}{2 m_{b}} \overrightarrow{\mathbf{L}}^{(b)}-\frac{1}{2 m_{a}} \overrightarrow{\mathbf{L}}^{(a)}\right] \cdot \vec{\theta}-\frac{Z e^{2}}{2 \mathbf{X}^{3}} \overrightarrow{\mathbf{E}} \cdot \vec{\theta}
$$

It is easy to see that this result reduces to the precedent one, if one considers the nucleus (particle "b") as localized at the origin and possessing an infinite mass.

Finally, in addition to the energy level shift at tree level for Hydrogen atom obtained in [3], there exists another term which takes into account the noncommutativity in the momentum sector of NCQPS in such a way that :

$$
\Delta E_{N C}^{H-a t o m}=-\frac{1}{2 m}\langle\overrightarrow{\mathbf{L}} \cdot \vec{\eta}\rangle=-\frac{1}{2 m}\left[\langle\overrightarrow{\mathbf{L}} \cdot \vec{\beta}\rangle+\left\langle\frac{Z e^{2}}{2 \mathbf{X}^{3}} \overrightarrow{\mathbf{L}} \cdot \vec{\theta}\right\rangle\right]
$$

\section{Conclusion}

In 4, it is emphasized that there is no noncommutative corrections at tree level for Hydrogen atom. Solving the NC two-body Schrödinger equation and considering that $\theta_{i j}$ changes the sign under charge conjugation, [8], the authors contradict the result obtained (and confirmed) in 3 .

In our work, following our approach, we have found that the kinetic term in the Hamiltonian gives an additional correction term to the result obtained in [3], (See (20)). Following our assumption which consists to say that the noncommutativity $\beta$ in the momentum sector of QPS is of the same magnitude as the noncommutativity $\theta$ in the space sector, up to a dimension factor depending on the physical system under study, we are tempted to deduce that effectively there is no noncommutative effects. We are motivated by the fact that $\vec{\beta} \sim-\vec{\theta}$ and by the presence 
of a coefficient of $\vec{\theta}$ in (20) (as well as in (111)), which is specific to the considered physical system. It seems that this may ensure the annihilation of the two terms, in such a way that the noncommutative effects do not really appear. The proof that noncommutativity exists really is already given by the simple example of free particle, which behaves like a harmonic oscillator with a (very small) Larmor frequency tied to $\beta$ (and so to $\theta$ ). It seems that it is the variation of the potential when it exists which contributes to the annihilation of this NC effect.

In another hand, it appears that noncommutativity is deeply tied to the presence of some magnetic sources in the space at scales near the planck one, and it is supposed to be a quantum effect of gravity, 9]. However, we think that this problem needs a deep analysis to be well understood.

Furthermore, in [10] an analog approach has been followed that consists to contruct an isotropic representation representing general transformations on NCQPS. Nevertheless, in this approach the authors have not used one important requirement on these transformations which leads to to the natural condition $\theta=-\beta$ we have obtained just by requiring an orthogonality property of these transformations. In fact, their intention being to work in full generality, they assumed that in general $\theta+\beta \neq 0$.

In future works, we plan to treat within our framework some other quantum examples like : NCQED, Bohm-Aharanov, Lamb shift , Stark, Zeeman and Hall effects,...

Acknowledgments : The authors would like to thank Abdus Salam ICTP, where this work was performed, for hospitality. A.E.F. Djemä̈ would also like to address his special thanks to Arab Fund and the Associateship scheme of ICTP for their support and help. The authors are also very indebted to A. Smailagic and E. Spallucci for useful discussions and for having shown us their work [10].

\section{References}

[1] J.E. Moyal : Proceedings of the Cambridge Philosophical Society 45 (1949)99 ; J. Vey : commentari Mathematici Helvetici 50 (1975)421

M. Flato \& al : Compositio Mathematica 31 (1975)47 ; M. Flato \& al : J. Math. Phys. 17 (1976)1754 ; F. Bayen \& al : Lett. Math. Phys. 1 (1977)521 ; F. Bayen \& al : Annals of Physics 111 (1978)61, 111

[2] A.E.F. Djemaï : Int. J. Theo. Phys. 35 (1996)519

[3] M. Chaichian \& al : Phys. Rev. Lett. 86(2001)2716 hep-th/0010175; Comments on the Hydrogen Atom in the Noncommutative Space hep-th/0212259

[4] P-M. Ho and H-C. Kao : Noncommutative Quantum Mechanics from Noncommutative Quantum Field Theory hep-th/0110191

[5] D. Kochan and M. Demetrian : QM on Noncommutative Plane hep-th/0102050

[6] C. Duval \& P.A. Horvathy : Phys. Lett. B479 (2000)284 [hep-th/002233]; J. Gamboa \& al : Phys. Rev. D64 (2001)267 hep-th0010220 ; V.P. Nair and A.P. Polychronakos : Phys. Lett. B 505 (2001)267 hep-th/0011172 ; B. Morariu and A.P. Polychronakos : Nucl. Phys.B 610 (2001)531 hep-th/0102157 ; A. Jellal : Orbital Magnetism of Two-dimension Noncommutative Confined System hep-th/0105303; C. Duval \& P.A. Horvathy : J. Phys. A34(2001)10097 hep-th/0106089 ; J. Gamboa \& al : Noncommutative Quantum Mechanics : The Two-dimensional Central Field hep-th/0106125 ; S. Bellucci \& al : Phys. Lett. B522 
(2001)345 hep-th/0106138; H.R. Christiansen and F.A. Schaposnik : Noncommutative Quantum Mechanics and Rotating Frames hep-th/0106181 ; R. Banerjee : Mod. Phys. Lett. A17 (2002)631 hep-th/0106280 ; C. Acatrinei : JHEP 0109 (2001)007 hep-th/0107078 ; P.A. Horvathy : Ann. Phys. 299 (2002)128 hep-th/0201007 ; S. Bellucci \& A. Nersessian : Phys. Lett. B 542 (2002)295 hep-th/0205024 ; A. Smailagic \& E. Spallucci : Feynman Path Integral on Noncommutative Plane hep-th/0307217 , UV Divergence-free QFT on Noncommutative Plane hep-th/0308193.

[7] A. Connes \& al : JHEP 9802 (1998)003 hep-th/9711162 ; M.R. Douglas and C.M. Hull : JHEP 9802 (1998)008 hep-th/9711165 ; C. Chu and P. Ho : Nucl. Phys. B550(1999)151 hep-th/9812219; Nucl. Phys. B568 (2000)447 hep-th/9906192 ; V. Schomerus : JHEP 9906 (1999)030 hep-th/9903205 ; N. Seiberg and E. Witten : JHEP 9909 (1999)032 hep-th/9908142 and references therein.

[8] M.M. Sheikh-Jabbari : Phys. Rev. Lett. 84 (2000)5265 hep-th/0001167

[9] H.S. Snyder : Phys. Rev. 71 (1946)38 ; C.N. yang : Phys. Rev. 72 (1947)874.

[10] A. Smailagic \& E. Spallucci : Phys. Rev. D65 (2002)107701 hep-th/0108216 , J. Phys. A35 (2002)L363 hep-th/0205242. 